УДК 531.793

\title{
Построение поверхностей постоянной средней кривизны
}

\author{
М.А. Чешкова ${ }^{1}$ И.В. Поликанова ${ }^{2}$ \\ ${ }^{1}$ Алтайский государственный университет (Барнаул, Россия) \\ ${ }^{2}$ Алтайский государственный педагогический университет (Барнаул, Россия)
}

\section{Construction of Surfaces with Constant Mean Curvature}

\author{
M.A. Cheshkova ${ }^{1}$, I.V. Polikanova ${ }^{2}$ \\ ${ }^{1}$ Altai State University (Barnaul, Russia) \\ ${ }^{2}$ Altai State Pedagogical University (Barnaul, Russia)
}

В данной работе исследуются поверхности постоянной средней кривизны (ПСК). Торы ПСК изучал Х. Вентье Позднее У. Абреш доказал, что торы Вентье имеют одно семейство плоских линий кривизны, и охарактеризовал их с помощью эллиптических интегралов. В работе А.И. Бобенко рассматривается задача построения торов ПСК в $E^{3}, S^{3}, H^{3}$. В этой работе исследуются поверхности вращения ПСК. Используя теорему Бонне о существовании поверхности ПСК, параллельной поверхности постоянной положительной гауссовой кривизны, для поверхностей вращения постоянной положительной гауссовой кривизны строятся поверхности постоянной средней кривизны. Доказано, что они являются также поверхностями вращения. Семейства плоских линий кривизны (меридианы) описаны с помощью эллиптических интегралов. Поверхности постоянной гауссовой кривизны также описаны с помощью эллиптических интегралов. С использованием специализированного программного обеспечения строятся рассматриваемые поверхности.

Ключевые слова: параллельная поверхность, средняя кривизна, гауссова кривизна, теорема Бонне, эллиптические интегралы.

DOI 10.14258/izvasu(2018)4-22

1. Введение. Пусть $M$ - гладкая поверхность в евклидовом пространстве $E^{3}, n-$ единичный вектор нормали. Определен оператор $A: \partial_{X} n=-A X$. Собственные значения $k_{1}, k_{2}$ оператора $A$ называются главными кривизнами поверхности, полусумма их $H$ $=12\left(k_{1}+k_{2}\right)$ есть средняя кривизна, а произведение $K=k_{1} k_{2}-$ гауссова кривизна поверхности [1, с. 36].

Имеет место теорема Пуассона - Лапласса [2, с. 12]: Предположим, что двумерная гладкая поверхность М в $E^{3}$ является границей раздела двух однородных сред, находящихся в равновесии. Пусть $P_{1}$,
The paper studies surfaces with constant mean curvature (CMC) $H$. If $H=0$ then the surfaces are minimal. CMC tori were studied by H. Wente. U. Abresz proved that Wente tori have one family of planar lines of curvature and characterized them with elliptic integrals.

A.I. Bobenko in his studies considered the problem of constructing CMC tori $E^{3}, S^{3}, H^{3}$. In this paper, CMC surfaces of revolution are investigated. For a surface in $E^{3}$ the Bonnet's theorem states that for any surface having constant positive Gaussian curvature, there exists a surface parallel to it with a constant mean curvature.

According to this statement, for surfaces of revolution with constant positive Gaussian curvature, CMC surfaces are constructed using the Bonnet's theorem. It is proved that constructed surfaces are also surfaces of revolution. A family of plane curvature lines (meridians) is described by elliptic integrals, and surfaces with Gaussian curvature are also described by elliptic integrals. These surfaces are constructed using the mathematical software package.

Key words: parallel surface, mean curvature, Gaussian curvature, Bonnet theorem, elliptic integrals.

$\mathrm{P}_{2}$ - давление в средах. Тогда средняя кривизна Н поверхности $M$ постоянна и равна $H=h\left(P_{1}-P_{2}\right)$, где постоянная $\lambda=\frac{1}{h}$ называется коэффициентом поверхностного натяжения.

Поверхности, для которых $H=$ const, называются поверхностями постоянной средней кривизны (ПСК). Если $H=0$, то поверхности минимальные.

Отрицательный ответ на проблему Хопфа [3]: Существуют ли компактные поверхности ПСК, отличные от сферы, получен при дополнительных предположениях [4], в частности, дана сле- 
дующая формулировка теоремы Хопфа : Единственная поверхность ПСК при $H=1 / 2$, топологически эквивалентная сфере, - это стандартная сфера радиуса 2.

Вентье [5] изучил торы ПСК. Дальнейшее изучение поверхностей ПСК продолжено в [6].

В работе Абреша [7] доказано, что торы Вентье имеют одно семейство плоских линий кривизны.

Поверхности ПСК в $E^{n}$ изучаются в монографии Чена [8]. Доказано, что если неминимальная в $E^{n} 2$-поверхность имеет параллельный вектор средней кривизны, то ([8], с. 10) это либо минимальная поверхность в $E^{n}$, либо минимальная поверхность на гиперсфере в $E^{n}$, либо 2-поверхность в $E^{3}$ с постоянной средней кривизной, либо 2поверхность на 3 -сфере $S^{3}$ в $E^{4}$ с постоянной средней кривизной.

Исследованию поверхностей постоянной кривизны в групповых пространствах посвящены работы Д.А. Бердинского [9] и J.F. Dorfinester, J-I. Inoguchi, S. Kobayashi, H. Wu. [10].

В.Т. Фоменко в работе [11] приводит условие, обобщающее условие Риччи, Нордена, Лоуссона, о изометрическом погружении двумерной метрики в трехмерное риманово пространство постоянной средней кривизны.

В работах В.И. Ильгисонис, А.А. Сковорода, Е.А. Сорокиной, И.А. Тайманова [12],[13] показано, что экстремаль объема, заключенного внутри ториодальной поверхности заданной площади, достигается поверхностью постоянной кривизны.

Поверхность $\bar{M}$ в $E^{3}$ называется параллельной поверхности $M$, если она состоит из концов отрезков постоянной длины, отложенных на нормалях поверхности $M$ от точек этой поверхности. Касательные плоскости в соответствующих точках будут параллельными.

Для поверхностей в $E^{3}$ имеет место обратная теорема Бонне Какова бъ ни была поверхность $M$, имеющая постоянную положительную гауссову кривизну, существует параллельная ей поверхность с постоянной средней кривизной.

Основная цель данной работы: построение поверхностей постоянной средней кривизны из поверхностей вращения постоянной положительной гауссовой кривизны.

2. Основные формулы. Пусть $r=$ $r(u, v)$ - уравнение поверхности $M, n-$ орт нормали, $h=$ const). Уравнение параллельной поверхности $\bar{r}$ имеет вид

$$
\bar{r}=r+h n .
$$

Обозначим через $K, H, \bar{K}, \bar{H}$ - гауссовы и средние кривизны поверхностей $M, \bar{M}$, соответственно. Имеем

$$
\bar{K}=\frac{K}{1-2 h H+h^{2} K}, \bar{H}=\frac{H-h K}{1-2 h H+h^{2} K} .
$$

Положим в (2)

$$
1-K h^{2}=0, h= \pm \frac{1}{\sqrt{K}} .
$$

Тогда

$$
\bar{H}= \pm \frac{\sqrt{K}}{2} .
$$

Это есть результат обратной теоремы Бонне. Он дает возможность построения поверхностей постоянной средней кривизны по поверхностям постоянной гауссовой кривизны.

Будем строить параллельные поверхности для поверхности вращения постоянной положительной гауссовой кривизны.

3. Поверхности постоянной средней кривизны. В евклидовом пространстве $E^{3}$ рассмотрим поверхность вращения $M$, полученную вращением плоской кривой вокруг оси. Обозначим через $k=(0,0,1)$ - орт оси, а через $e=(\cos (v), \sin (v), 0)$ - радиус-вектор единичной окружности, расположенной в плоскости, ортогональной оси.

Тогда поверхность $M$ можно задать в виде

$$
r=u e(v)+f(u) k,
$$

где $f=f(u)$ - дифференцируемая функция, $u, v$ - параметры.

Обозначим через $n$ - орт нормали к поверхности $M$. Тогда

$$
n=\frac{f(u)^{\prime} e(v)-k}{\sqrt{\left(f(u)^{\prime}\right)^{2}+1}} .
$$

Главные кривизны $k_{1}, k_{2}$ поверхности $M$ имеют вид

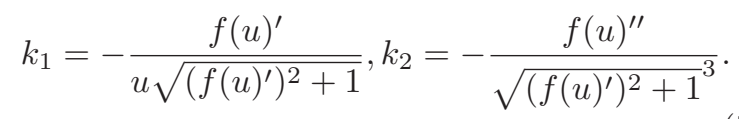

Имеем дифференциальное уравнение

$$
\frac{f(u)^{\prime}}{u{\sqrt{\left(f(u)^{\prime}\right)^{2}+1}}^{\left({ }^{\left(f(u)^{\prime}\right)^{2}+1}\right.}}=\frac{f(u)^{\prime \prime}}{}=K .
$$

Получим решения

$$
f(u)= \pm \int_{0}^{u} \sqrt{\frac{K t^{2}-(c-1)}{c-K t^{2}}} d t, c=\text { const. }
$$

Имеем

$$
f(u)= \pm \frac{I \sqrt{c-1} \text { EllipticE }\left(\frac{u \sqrt{K c}}{c}, \frac{\sqrt{(c-1) c}}{c-1}\right)}{\sqrt{K}}+c_{1},
$$

c, $c_{1}=$ const .

Для определенности, полагаем $K=1$. 
Имеем

$$
\begin{aligned}
& f(u)= \pm \int_{0}^{u} \sqrt{\frac{t^{2}-(c-1)}{c-t^{2}}} d t+c_{1}, c, c_{1}=\text { const } \\
& f(u)= \pm I \sqrt{c-1} \text { EllipticE }\left(\frac{u}{\sqrt{c}}, \frac{\sqrt{(c-1) c}}{c-1}\right)+c_{1} .
\end{aligned}
$$

Полагая $c=1 / 2$, получим

$$
f(u)= \pm \frac{\sqrt{2}}{2} \operatorname{EllipticE}(u \sqrt{2}, I)+c_{1} .
$$

Построение поверхности вращения постоянной гауссовой кривизны рассматривается в [14].

Рассмотрим поверхности вращения $M_{1}, M_{2}$ при $c_{1}=0$ и $c_{2}=\frac{2}{\sqrt{2}} \operatorname{EllipticE}\left(\frac{1}{\sqrt{2}}, I\right), u \in$ $[0, \sqrt{c}], v \in[-\pi, \pi]$.

Имеем

$$
\begin{aligned}
M_{1}: r(u, v)= & \left(u \cos (v), u \sin (v),+\frac{\sqrt{2}}{2} \operatorname{EllipticE}(u \sqrt{2}, I)\right), \\
M_{2}: r(u, v)= & \left(u \cos (v), u \sin (v),-\frac{\sqrt{2}}{2} \operatorname{EllipticE}(u \sqrt{2}, I)\right. \\
& \left.+\frac{2}{\sqrt{2}} \operatorname{EllipticE}\left(\frac{1}{\sqrt{2}}, I\right)\right) .
\end{aligned}
$$

Используя математический пакет, построим поверхности $M_{1}, M_{2}$ (рис. 1$)$.

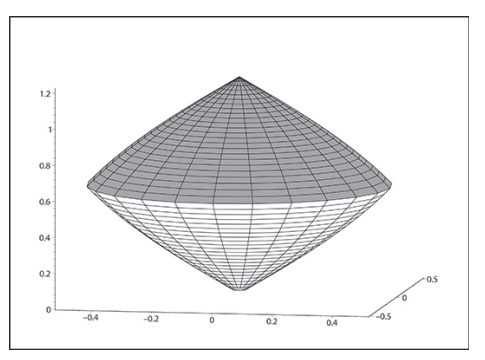

Рис. 1. Поверхности $M_{1}, M_{2}$

Полагаем в (1) $h=1$, а в (4) подставим функции $f=f(u)$ из $(9)$, получим

$$
f^{\prime}(u)= \pm \sqrt{\frac{2 u^{2}+1}{1-2 u^{2}}},
$$

$n= \pm \frac{1}{\sqrt{2}}\left(\sqrt{2 u^{2}+1} \cos (v), \sqrt{2 u^{2}+1} \sin (v),-\sqrt{1-2 u^{2}}\right)$.

Определим поверхности $\bar{M}_{1}, \bar{M}_{2}$, параллельные $M_{1}, M_{2}$ соответственно.

$$
\begin{gathered}
\bar{M}_{1}: r(u, v)=\left(u \cos (v)+\frac{1}{\sqrt{2}} \sqrt{2 u^{2}+1} \cos (v),\right. \\
u \sin (v)+\frac{1}{\sqrt{2}} \sqrt{2 u^{2}+1} \sin (v),+\frac{\sqrt{2}}{2} \operatorname{EllipticE}(u \sqrt{2}, I)- \\
\left.\frac{1}{\sqrt{2}} \sqrt{1-2 u^{2}}\right),
\end{gathered}
$$

$\bar{M}_{2}: r(u, v)=\left(u \cos (v)+\frac{1}{\sqrt{2}} \sqrt{2 u^{2}+1} \cos (v), u \sin (v)+\right.$ $\frac{1}{\sqrt{2}} \sqrt{2 u^{2}+1} \sin (v),-\frac{\sqrt{2}}{2} \operatorname{EllipticE}(u \sqrt{2}, I)+$ $\left.\frac{2}{\sqrt{2}} \operatorname{EllipticE}\left(\frac{1}{\sqrt{2}}, I\right)-\frac{1}{\sqrt{2}} \sqrt{1-2 u^{2}}\right)$.

Построим их (рис. 2).

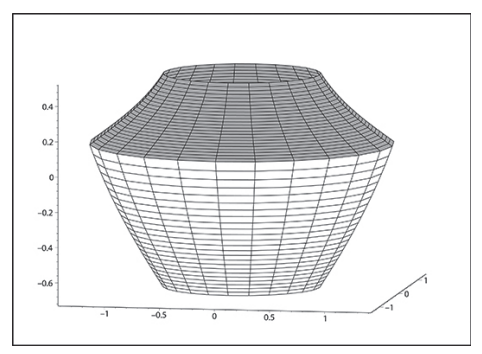

Рис. 2. Поверхности $\bar{M}_{1}, \bar{M}_{2}$

Рассмотрим еще один вариант поверхностей $\bar{M}_{1}^{*}, \bar{M}_{2}^{*}$ :

$$
\begin{gathered}
\bar{M}_{1}^{*}: r(u, v)=\left(u \cos (v)-\frac{1}{\sqrt{2}} \sqrt{2 u^{2}+1} \cos (v),\right. \\
u \sin (v)-\frac{1}{\sqrt{2}} \sqrt{2 u^{2}+1} \sin (v), \\
\left.+\frac{\sqrt{2}}{2} \operatorname{EllipticE}(u \sqrt{2}, I)+\frac{1}{\sqrt{2}} \sqrt{1-2 u^{2}}\right) . \\
\bar{M}_{2}^{*}: r(u, v)=\left(u \cos (v)-\frac{1}{\sqrt{2}} \sqrt{2 u^{2}+1} \cos (v),\right.
\end{gathered}
$$

$u \sin (v)-\frac{1}{\sqrt{2}} \sqrt{2 u^{2}+1} \sin (v),-\frac{\sqrt{2}}{2} \operatorname{EllipticE}(u \sqrt{2}, I)$

$$
\left.+\frac{2}{\sqrt{2}} \operatorname{EllipticE}\left(\frac{1}{\sqrt{2}}, I\right)+\frac{1}{\sqrt{2}} \sqrt{1-2 u^{2}}\right) .
$$

Построим их (рис. 3). 
Из данных утверждений следует

Теорема. Поверхности $\bar{M}_{!}, \bar{M}_{2}, \bar{M}_{1}^{*}, \bar{M}_{2}^{*}$ есть поверхности врашения, у которых плоские меридианы имеют вид

$$
\begin{gathered}
R=\left(u+\frac{1}{\sqrt{2}} \sqrt{2 u^{2}+1},\right. \\
\left.+\frac{\sqrt{2}}{2} \text { EllipticE }(u \sqrt{2}, I)-\frac{1}{\sqrt{2}} \sqrt{1-2 u^{2}}\right), \\
R=\left(u+\frac{1}{\sqrt{2}} \sqrt{2 u^{2}+1},\right. \\
-\frac{\sqrt{2}}{2} \text { EllipticE }(u \sqrt{2}, I)+\frac{2}{\sqrt{2}} \operatorname{EllipticE}\left(\frac{1}{\sqrt{2}}, I\right)- \\
\left.\frac{1}{\sqrt{2}} \sqrt{1-2 u^{2}}\right), \\
R=\left(u-\frac{1}{\sqrt{2}} \sqrt{2 u^{2}+1},\right.
\end{gathered}
$$

$$
\begin{gathered}
\left.-\frac{\sqrt{2}}{2} \operatorname{EllipticE}(u \sqrt{2}, I)+\frac{1}{\sqrt{2}} \sqrt{1-2 u^{2}}\right), \\
R=\left(u-\frac{1}{\sqrt{2}} \sqrt{2 u^{2}+1}, \frac{\sqrt{2}}{2} \operatorname{EllipticE}(u \sqrt{2}, I)+\right. \\
\left.\frac{2}{\sqrt{2}} \operatorname{EllipticE}\left(\frac{1}{\sqrt{2}}, I\right)+\frac{1}{\sqrt{2}} \sqrt{1-2 u^{2}}\right),
\end{gathered}
$$

соответственно.

4. Заключение. Основным результатои работы является разработка алгоритма построения поверхностей вращения постоянной средней кривизны из поверхностей вращения постоянной гауссовой кривизны. Кроме того, предложенная работа позволяет моделировать поверхности вращения постоянной средней кривизны, используя эллиптические интегралы.

\section{Библиографический список}

1. Кобаяси Ш., Номидзу К. Основы дифференциальной геометрии. - М., 1981.

2. Тужилин А.А., Фоменко А.Т. Элементы геометрии и топологии минимальных поверхностей. - М., 1991.

3. Hopf H. Differential geometry in the larle. Lect. Notes Math. - V. 1000. - Berlin Heiderberg - Ney York - Tokyo - Springer, 1986.

4. Бобенко А.И. Поверхности постоянной средней кривизны и интегрируемые уравнения // УМН. - 1991. - Т. 6. - Вып. 4 (280).

5. Wente H. Counterexample to conjecture of H. Hopf. Pacific J. Math. - 1986. - № 121.

6. Wente H. Constant mean curvature of annular type // Calculus of Variations and Partial Differential Equations. - 2002. - T. 14. — № 2.

7. Abresch U. Constant mean curvature tori in elliptic function // J.reine $u$. angew. Math. 1987. - Bd. 304.

8. Chen B. Geometry of submanifolds and its applications. - Tokyo, 1981.
9. Бердинский Д.А. О поверхностях постоянной средней кривизны в группах гейзенберга // Мат. труды. - 2010. - Т. 13. - № 2 .

10. Dorfinester J.F., Inoguchi J-I., Kobayashi S., $\mathrm{Wu}$ H. Constant mean curvanure in hyperbolic 3space via loop groops // Journal fur die reine und angewandte mathematik. - 2014. - № 686 .

11. Фоменко В.Т. О метриках, возникающих на поверхностях постоянной средней кривизны // Известия вузов. - 2004. - № 10.

12. Ильгисонис В.И., Сковорода А.А., Сорокина Е.А. О тороидальных поверхностях вращения с постоянной средней кривизны // Вопросы атомной науки и техники. Серия: Термоядерный синтез. - 2016. - Т. 39. - № 1 .

13. Сковорода А.А., Тайманов И.А. О значении средней кривизны в геометрии магнитного поля ловушек для удержания плазмы // Abpbrf gkfpva. - 2010. - № 36 .

14. Чешкова М.А. Построение поверхности вращения постоянной гауссовой кривизны // Сборник трудов Всероссийской конференции по математике. - Барнаул, 2017. 